\title{
Primary Care Physician Designation and Response to Clinical Decision Support Reminders
}

\section{A Cross-Sectional Study}

Jeffrey M. Weinfeld'; Paul N. Gorman²

'Department of Family Medicine, Georgetown University School of Medicine, Washington, DC;

${ }^{2}$ Department of Medical Informatics and Clinical Epidemiology, Oregon Health \& Science University, Portland, Oregon

\section{Keywords}

Primary health care, clinical decision support systems, electronic health records

\section{Summary}

Objective: Clinical decision support (CDS) has been shown to improve process outcomes, but overalerting may not produce incremental benefits. We analyzed providers' response to preventive care reminders to determine if reminder response rates varied when a primary care provider (PCP) saw their own patients as compared with a partner's patients. Secondary objectives were to describe variation in PCP identification in the electronic health record (EHR) across sites, and to determine its accuracy.

Methods: We retrospectively analyzed response to preventive care reminders during visits to outpatient primary care sites over a three-month period where an EHR was used. Data on clinician requests for reminders, viewing of preventive care reminders, and response rates were stratified by whether the patient visited their own PCP, the PCP's partner, or where no PCP was listed in the EHR. We calculated the proportion of PCP identification across sites and agreement of identified PCP with an external standard.

Results: Of 84,937 visits, 58,482 (68.9\%) were with the PCP, 10,259 (12.1\%) were with the PCP's partner, and 16,196 (19.1\%) had no listed PCP. Compared with PCP partner visits, visits with the patient's PCP were associated with more requested reminders (30.9\% vs $22.9 \%)$, viewed reminders ( $29.7 \%$ vs $20.7 \%$ ), and responses to reminders ( $28.7 \%$ vs $12.6 \%$ ), all comparisons $p<0.001$. Visits with no listed PCP had the lowest rates of requests, views, and responses. There was good agreement between the EHR-listed PCP and the provider seen for a plurality of visits over the last year ( $\mathrm{K}=0.917)$.

Conclusions: A PCP relationship during a visit was associated with higher use of preventive care reminders and a lack of PCP was associated with lower use of CDS. Targeting reminders to the PCP may be desirable, but further studies are needed to determine which strategy achieves better patient care outcomes.

\section{Correspondence to:}

Jeffrey M. Weinfeld, MD, MBI,

3900 Reservoir Rd, NW, GM4B Pre-Clinical Sciences Building

Washington, DC 20009

Email: Jeff.Weinfeld@georgetown.edu

\author{
Appl Clin Inform 2016; 7: 248-259 \\ http://dx.doi.org/10.4338/ACl-2015-10-RA-0142 \\ received: November 3, 2015 \\ accepted: February 7, 2016 \\ published: April 27, 2016 \\ Citation: Weinfeld JM, Gorman PN. Primary care phys- \\ ician designation and response to clinical decision \\ support reminders: A cross-sectional study. Appl Clin \\ Inform 2016; 7: 248-259 \\ http://dx.doi.org/10.4338/ACl-2015-10-RA-0142
}




\section{Introduction}

The provision of primary medical care is associated with an increased life span, fewer lower birthweight babies, and lower all-cause mortality [1]. These benefits accrue from a primary care provider (PCP) seeing the same panel of patients over time. Identification of this panel is important for continuity of care, care coordination, and evaluating the cost, quality, and patient experience that a single provider delivers [2].

Clinical decision support (CDS) provides clinicians with knowledge and information at a relevant time to improve patient care [3]. Extensive research supports improved processes of care in general [4], in primary care settings [5], for preventive care [6], and in quality of care for diabetes and heart disease [7]. A systematic review of the impact of CDS on process and outcomes of care reported a median improvement of $4.2 \%$ [8].

While CDS typically presents reminders or alerts to whomever is using the electronic health record (EHR), in group practice settings many providers use the same record for different purposes and types of visits. For example, reminders for preventive care may not be seen as relevant when a patient is being seen by the PCP for an acute problem or when a patient is being seen by a PCP's partner for acute illness care. Presenting the same reminders to all providers at all visits may contribute to inappropriately ignored or overridden alerts, so called "alert fatigue" [9] and result in reduced response rates. Despite the value of CDS, its low effectiveness remains a challenge [10] with override rates for medication-related CDS as high as 49-96\% [9]. It is easy to see how PCPs can be overwhelmed: one study found that PCPs were presented with an average of 56 asynchronous alerts per day - in addition to drug-related alerts - and spent 49 minutes on average per provider responding to them [11]. However, there could be an incremental benefit in showing a reminder to every provider, regardless of who is seeing the patient, resulting in additional appropriate care. Providers have indicated a desire for targeted alerting and the ability to indicate user preferences [12]. EHRs may attempt to target CDS by specialty, but to remind only the PCP, the PCP must be accurately identified in the EHR.

\subsection{Objectives}

Given these issues for CDS in a primary care setting, we undertook this study to understand PCP identification in the EHR, assess the accuracy of PCP identification, and determine whether response to preventive reminders varies with the provider's relationship to the patient during a visit.

\section{Methods}

\subsection{Design and Inclusion Criteria}

We conducted a cross-sectional analysis of adult outpatient visits to PCPs at primary care sites. Visits by patients over 18 years of age to PCPs in Internal Medicine, Family Medicine, Internal MedicinePediatrics or student health (largely Family Medicine) over a 3-month period in late 2013 were included. Visits to resident physicians were excluded.

\subsection{Setting}

Visits occurred in outpatient primary care practices that are owned or operated by MedStar Health, a not-for profit health system in the metropolitan Baltimore, MD and Washington, DC area with 5600 physicians and over 2 million total outpatient visits (including emergency room visits) annually at the time the study was conducted [13]. The organization shared a common EHR for outpatient sites during the time of the study.

A third-party decision support system was installed which displayed a yellow alert button indicating that reminders for the patient were available. Providers had to click this button to view reminders and take action. 
All preventive reminders displayed by the system were included in the study. Those included reminders for vaccines (influenza and pneumococcal), preventive care (pap smears, mammography, HIV screening), and cardiovascular prevention (Million Hearts Initiative [14] which included aspirin, blood pressure control, lipid management, and smoking). Responses to medication alerts were tracked and displayed by a different system and were not included.

\subsection{Data Extraction, Definitions and Analysis}

EHR data was obtained by querying an internal data warehouse using a vendor-licensed data extraction tool, which received regular data extracts from the EHR. Research data was analyzed with SPSS 21 (IBM Software). Data on visits from October to December 2013 were included and queries were run between January and March 2014. Demographic data about patients and PCPs, site of care, and decision support data were collected for these visits.

We defined the EHR PCP during a visit as the PCP listed in the EHR for the patient at the time of the query. The "provider seeing the patient" was defined as the provider responsible for the visit note. "PCP visits" were defined when the EHR PCP was the same as the provider responsible for the note. "Non-PCP visits" were defined as those where the EHR PCP and the provider responsible for the note did not match. Finally, "No-PCP visits" where identified when no provider was listed in the EHR as PCP. Providers were matched by using the National Provider Identifier (NPI) number.

We assessed the accuracy of the PCP listed in the EHR using the plurality PCP method as described by Pham et al. [15]. This method assigns as PCP the provider who saw the patient for a plurality of visits over the prior year. Therefore the provider for visits during the year leading up to the 3 months of visits was also extracted.

The EHR automatically tracked clinician behavior in response to preventive care reminders. A "request" was recorded when a user clicked the reminder button requesting a list of reminders that were due. A "view" was recorded when a user viewed an individual reminder in detail. A "response" was recorded when a user responded to a reminder. A response could include carrying out the recommended action, test ordering, recording a refusal, or indicating that the test was previously performed.

\subsection{Outcomes and Statistical analysis}

Since the outcome of interest was response to reminders during visits, the visit was used as the unit of analysis rather than the patient. The primary outcome for the study was the unadjusted rate of reminder response during a PCP visit as compared with a non-PCP visit. An a priori sample size calculation using $\alpha=0.05$ and $\beta=0.80$ for the primary outcome found that 1094 visits needed to be included in each of these two groups.

Secondary outcomes included rates of reminder requesting and viewing for PCP visits, non-PCP visits, and no-PCP visits. Proportions of response types were compared using the Chi-square statistic. For PCP accuracy, we compared the patient's PCP as listed in the EHR with the PCP for the patient by plurality method using the kappa statistic (note that this is by patient not by visit, since a patient makes multiple visits but can only have one PCP and one plurality provider).

We calculated the number needed to get one reminder response for each PCP identified (instead of conducting a no-PCP visit) as 1/(PCP group visit response rate - no-PCP visit response rate). The number needed to get one reminder response for each visit scheduled with the PCP (instead of PCP partner) was calculated as 1/(PCP group visit response rate - non-PCP visit response rate).

The MedStar Health Research Institute Institutional Review Board reviewed and approved the study. 


\section{Results}

\subsection{PCP Identification}

A study flow diagram is shown in Figure 1. 84,937 non-resident visits to primary care sites occurred during the three-month time frame. For $80.9 \%$ of the visits, a PCP was identified, and for $19.1 \%$, no PCP was identified. Of all visits, $68.9 \%$ were to the patient's PCP and $12.1 \%$ were not with the PCP.

Providers for visits were predominantly physicians $(89.4 \%)$ in primary care specialties including Internal Medicine (77.6\%), Family Medicine (17.3\%), and Medicine-Pediatrics (1.06\%). The remaining $4.1 \%$ were classified as "Student Health" ( Table 1). The age distribution of patients was bimodal, with a median age of 56 , and a smaller peak at 28 years (data not shown). During the threemonth study period, 67,805 patients made one or more visits. There were 505 unique PCPs identified during these visits of which $90.9 \%$ were physicians ( $>$ Appendix 1 ). Internal Medicine was the most common specialty for PCPs (80.1\%) followed by Family Medicine (18.7\%).

\subsection{Accuracy of PCP identification in the EHR}

We calculated the PCP for each patient for the prior year by the plurality method and compared the result to the PCP listed in the EHR at the time of data extraction. For the 50,778 unique patients where both a PCP and plurality provider could be established, the kappa for agreement between listed PCP and calculated plurality PCP was 0.917 ( $\mathrm{p}<0.001, \mathrm{SE} \pm 0.001)$.

The percent of visits where a PCP was listed in the chart varied widely among the 62 patient-care sites ( $>$ Appendix 2). The median value for percent of visits to the EHR-identified PCP was $95.1 \%$ of visits, however the interquartile range was 67.7 - 99.1\%. A few sites rarely identified PCPs, but most sites typically identified one. The continuity of care as measured by the percent of visits to the PCP also varied widely. Again, there was a large range, but the median value among sites was $80.3 \%$ (interquartile range $35.7 \%-90.9 \%$ ). Both of these distributions were negatively skewed with a tail at the lower percentages.

\subsection{Provider Response to Reminders}

Analysis of provider response to reminders was limited to those visits where the provider responsible for the document was a provider with a NPI number, which included physicians assistants, nurse practitioners, and physicians. With this added constraint, 84,907 visits were eligible ( Table 2). Providers requested decision support reminders in $25.2 \%$ of these visits, viewed the reminder in detail in $24.3 \%$, and responded with some action in $23.5 \%$.

For the primary outcome of reminder response rate, providers responded to reminders in $28.7 \%$ of PCP visits compared with $12.6 \%$ of non-PCP visits $(\mathrm{p}<0.001)$. This pattern was consistent for secondary outcome behaviors: provider requesting, viewing, and taking action in response to the reminder were all significantly different across the three visit types $(\mathrm{p}<0.001)$. Providers were significantly more likely to request, view, and respond to reminders during PCP visits as compared with non-PCP visits ( $\mathrm{p}<0.001$ for each of the three pairwise comparisons). These findings are illustrated in $>$ Figure 2.

If a PCP were to be identified for a patient before a no-PCP visit - effectively switching the visit groups - we would expect that the reminder response rate would improve to those seen in the PCP group. The absolute increase in reminder response from adding a PCP for each no-PCP visit found in the study was $21.7 \%$. We can therefore calculate a "number needed to treat:" 4.6 PCPs would need to be identified before no-PCP visits to gain one additional reminder response (for the three-month period). Similarly, by improving continuity of care, and scheduling non-PCP visits with the PCP, the absolute increase in reminder response would be $16.1 \%$. The number of patients that would need to be scheduled with the PCP instead of a non-PCP for each reminder response is 6.2. 


\section{Discussion}

\subsection{Main findings}

There are three main findings arising out of this analysis. First, the vast majority of patient records in the EHR system list a primary care provider (95\%), and deficiencies in this regard are primarily by practice site. Second, the listed primary care provider is largely accurate when compared to a calculation based on a plurality of visits in the prior year. Third, reminders were far more likely to prompt a response by the provider when delivered during a visit with the patient's primary care provider than when delivered during a visit with another provider.

\subsection{Interpretation in the context of published literature}

There is a body of literature suggesting that patient care outcomes are improved by continuity of care and primary care [1] but this literature was published before widespread EHR adoption, and so does not examine improved care and CDS. There are very few contemporary studies that examine primary care and CDS, but the present study is consistent with what has been reported previously. Ferrante et al found that receipt of preventive care services was associated both with having a personal physician and with the use of CDS tools, but did not connect primary care directly with CDS [16]. The median increase in process improvement found in a systematic review of CDS [8] is consistent with the difference between the groups found in our study. Other studies have also identified physician variation in CDS use. For example, studies found that not every physician makes use of every EHR feature, and that primary care, patient-centeredness, and larger practice size were factors predicting use [17] as were physicians' uncertainty beliefs [18]. And while imaging ordering increases in practices with an EHR, prior doctor visits with the patient (continuity of care) reduced the odds of imaging ordering [19].

\subsection{Discussion of Findings}

It is not clear why some sites in the system have much lower percent of their patients with a listed PCP. It is possible that more urgent care visits, a higher turnover of patients, providers, or staff, weaker workflow procedures, or any number of patient characteristics may result in lower PCP identification. Stronger office and institutional policies and improved workflows surrounding PCP identification may be possible remedies.

Inaccuracies in PCP identification are likely to occur when the patient changes the provider in a practice who is acting as PCP. It seems likely that some inaccuracy with PCP turnover is inevitable. Lack of any PCP identification was higher than expected and may be due to oversight in PCP identification. Given our results, efforts should be made to verify and update PCPs at each visit.

Higher PCP response to reminders during PCP visits may be attributed to a variety of factors. A key factor is likely to be relevance to the visit and the type of visit, for example preventive, acute care, chronic care. Primary care visits to the PCP typically address preventive care to some degree regardless of chief complaint, so reminders on preventive topics may have been sought and used. Another possibility is that non-PCP visits (ie to PCP partners) are more often for acute illness or episodic visits where preventive care is a less critical part of the agenda. Patient expectations during non-PCP visits may not include preventive care, resulting in less provider use of reminders, or time pressures may be higher in these visits.

\subsection{Implications for Practices and Organizations}

With widespread adoption of primary care medical homes, care coordination, and quality measurement, health care organizations will depend upon accurate identification of the provider who is responsible for each primary care patient. Clear and complete PCP identification will allow attribution of quality measures to the PCP, making them more realistic and valid, rather than attributing measures to the last provider who saw the patient. An enterprise policy and recommended workflow for PCP identification in the EHR would be advisable. 
When listed in the EHR, the PCP provided was shown to be accurate when compared to an external standard. This agreement suggests that the usual workflow for identifying a PCP during an office is sufficient. It is important to note that our methodology does not compare the chart PCP to a gold standard (such as asking the patient to identify their PCP), but instead to a standard method of identifying a PCP. If the PCP is not listed in the EHR, the simplicity of the plurality PCP method could quickly be used to identify one.

Organizations may conclude that since PCPs seeing their own patients are the primary reminder users, that increased targeting of reminders to the PCP during PCP visits is warranted in order to reduce over alerting during no- and non-PCP visits. Conversely, organizations may decide that the small additional amount of reminder response that is provided during non-PCP and no PCP visits may be beneficial and should be encouraged to take advantage of additional quality opportunities. Targeting of reminders to PCPs could be tuned such that while most reminders go to the PCP, perhaps time critical or specific reminders might go to everyone.

\subsection{Informatics Implications}

While we did find differences in reminder responses, we do not know if these were irrelevant or inappropriately ignored, and thus we do not know whether they would constitute alert fatigue. Additional study of the role of PCP relationship and type of visit in alert fatigue would be valuable.

We also do not know the optimal level of reminding for various types of visits or providers; the levels found may be appropriate even though they differ by provider relationship. The authors do not take a position as to whether the observed level of reminding was optimal or not.

If organizations chose to target reminders, EHR systems would need certain capabilities. For example, the PCP and the function of visit (acute vs. chronic care) would need to be saved as computable fields. The CDS implementer would need to specify which type of user is the target for the reminder (PCP vs. any provider). While the PCP is identified in most EHR implementations, the availability of these other fields within CDS systems is likely variable.

\subsection{Limitations}

There are several important limitations of this study. First, the work was done in one organization and set in adult primary care specialties, so findings may not generalize to other care settings, EHRs, or specialties. Reminders examined were for preventive and ongoing care and had to be requested by the treating provider, and did not include other CDS such as medication alerts. An observational study design was used, so the association of provider continuity with higher reminder response rates cannot be assumed to be causal.

The fact that the PCP for a given visit could only be determined at the time of the query limits the accuracy of the study. It is possible that the PCP was changed between the time of the visit and the query. However, there was a limited period of time between the visit and the query, and we have no reason to expect net switching in a particular direction which would bias the study.

There are likely clustering effects within our data for which we have not adjusted. For example, a given physician or site may use CDS more or less frequently. Some doctors or sites may have greater or lower continuity of care, and some sites or doctors use the PCP listing more or less frequently. Given the straightforward outcome measures, we have not adjusted for these or other confounders in calculating response rates. It will be important to understand if findings can be explained by confounding factors such as visit purpose (acute illness vs. preventive care). A future prospective study could better account for clustering, explore factors that inhibit or increase CDS use, and account for confounding variables. Since the study was cross-sectional, we do not directly measure whether a reminder was presented to the same provider multiple times, so alert fatigue was not directly measured. An interventional design could examine if targeting PCPs with CDS leads to better reminder response then reminding all providers, and establish if it is a causal factor in CDS use. A qualitative or mixed-methods design would also be helpful to understand why PCPs do or do not use CDS during a given visit. 


\subsection{Conclusion}

We found that PCPs were identified in the EHR for $80.9 \%$ of visits and that there was variation in PCP identification by site. Since the accuracy of PCP identification in the EHR was high, typical workflows may be sufficient to identify a PCP. If the PCP is not listed, the feasibility of the plurality PCP method makes it a potential method for proposing a candidate PCP.

Finally, there were differences in how providers responded to decision support depending on their apparent relationship with the patient. Primary care physicians seeing their own patients requested more alerts, viewed more alerts, and responded to alerts more frequently than PCPs not seeing their patients, or when no PCP was identified. This may reflect the special relationship between primary care physicians and their patients, and point to one mechanism by which primary care improves health. Clinicians and managers may choose to use this information to improve primary care, and CDS implementers could use it to target alerts more effectively if desired.

\section{Clinical Relevance Statement}

In a multi-specialty group, we found that reminder response rates to PCPs were highest when PCPs were seeing their own patients, lower when seeing a partner's patients, and lowest when no PCP was identified in the chart. While optimal reminder response rates for these situations are not known, organizations may improve PCP identification in the EHR and reduce non-PCP visits to maximize reminder response rates. Further research is warranted to identify if this relationship is causal and the role of PCP non-targeted reminders in alert fatigue.

\section{Conflict of Interest Statement}

Neither author has any conflicts of interest.

\section{Protection of Human Subjects}

The study was performed in compliance with the World Medical Association Declaration of Helsinki on Ethical Principles for Medical Research Involving Human Subjects, and was reviewed by the MedStar Health Research Institute Institutional Review Board.

\section{Acknowledgments}

We would like to thank Peter Basch and John Janas for expert advice and insight into the workings of the EHR.

\section{Funding Support \\ None}

\section{Prior Publication}

Submitted as Masters Capstone to Oregon Health \& Science University without publication. Poster presented at internal MedStar Health Research Symposium 2015 without online publication

\section{Abbreviations}

primary care physician (PCP), clinical decision support (CDS), electronic health record (EHR), National Provider Identifier (NPI)

\section{Contributorship Statement}

Both authors jointly conceived of the project and study design, research and statistics carried out by JW with supervision from PG, paper drafted by JW, with editing and by PG. 


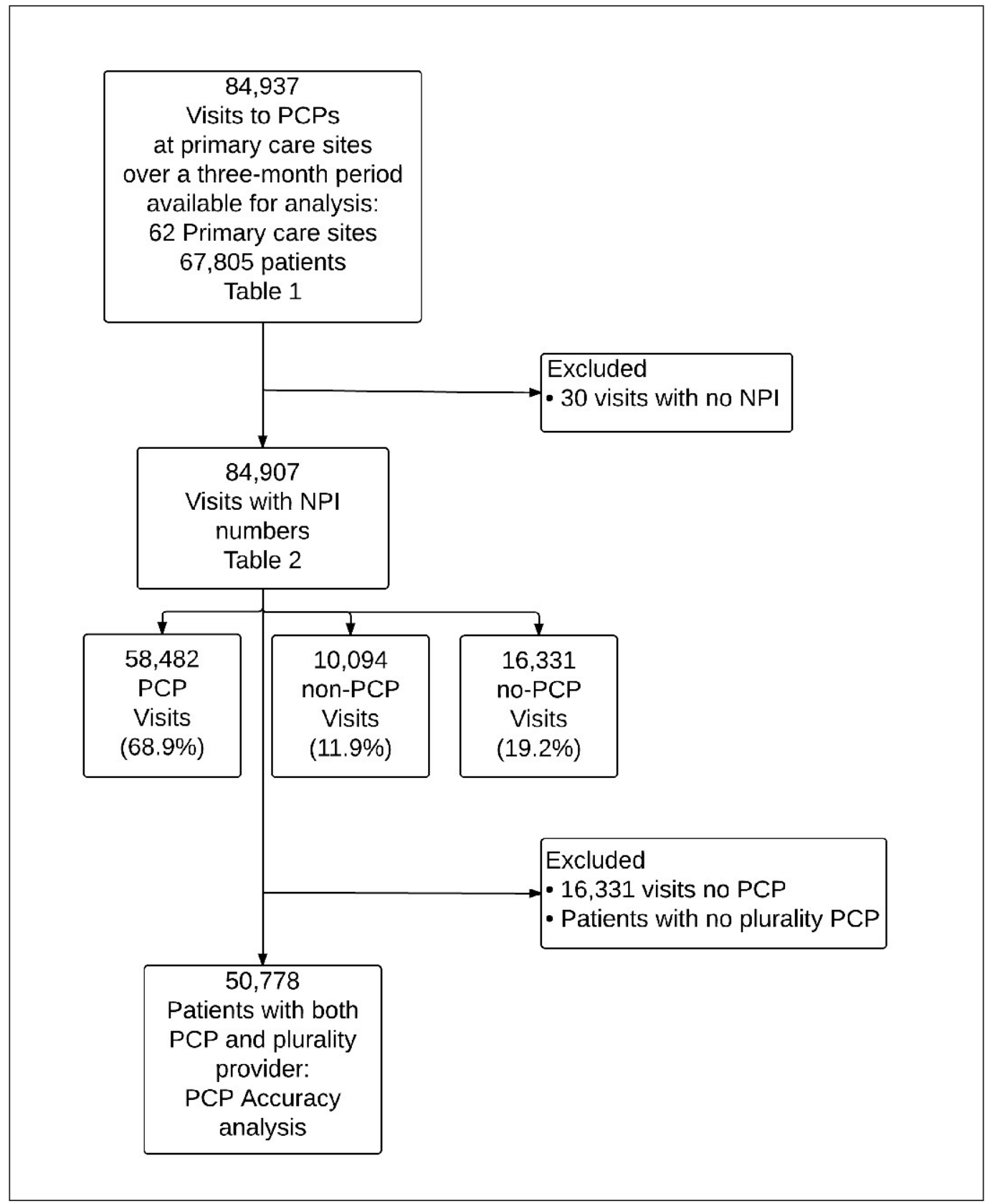

Fig. 1 Study Flow Diagram 


\section{Research Article}

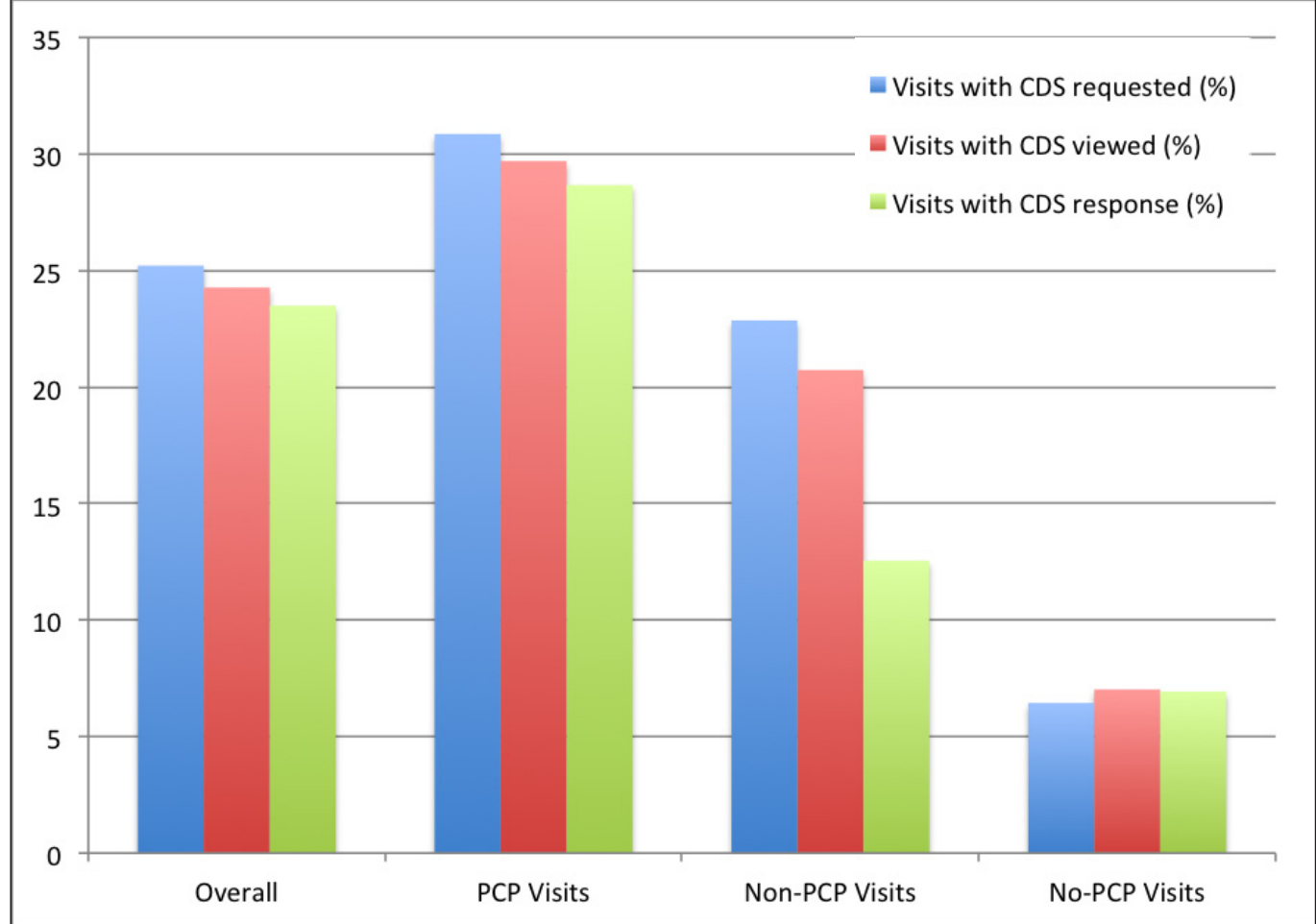

Fig. 2 Bar graph showing CDS behavior overall, during PCP, Non-PCP and No-PCP visits. 
Table 1 Provider Degree and Specialty for Visits $(n=84,937)$

\begin{tabular}{|c|c|c|}
\hline Characteristics & Visits & Percent \\
\hline \multicolumn{3}{|l|}{ Provider degree } \\
\hline Physician & 75,920 & 89.384 \\
\hline Nurse Practitioner & 6,328 & 7.450 \\
\hline Physician Assistant & 2,659 & 3.131 \\
\hline Other & 30 & 0.036 \\
\hline \multicolumn{3}{|l|}{ Provider specialty } \\
\hline Family Medicine & 7,839 & 17.34 \\
\hline Internal Medicine & 65,879 & 77.56 \\
\hline Medicine-Pediatrics & 899 & 1.06 \\
\hline Student Health & 3,435 & 4.04 \\
\hline
\end{tabular}

Table 2 Provider CDS behavior when conducting PCP visits, Non-PCP visits, and No-PCP visits

\begin{tabular}{|l|l|l|l|l|}
\hline Characteristic & $\begin{array}{l}\text { Total } \\
\text { Visits } \\
(\mathbf{n = 8 4 , 9 0 7 )}\end{array}$ & $\begin{array}{l}\text { PCP } \\
\text { visits } \\
(\mathbf{n = 5 8 , 4 8 2})\end{array}$ & $\begin{array}{l}\text { Non-PCP } \\
\text { visits } \\
(\mathbf{n}=10,094)\end{array}$ & $\begin{array}{l}\text { No-PCP } \\
\text { visits } \\
\mathbf{( n = 1 6 , 3 3 1 )}\end{array}$ \\
\hline Visits (\%) & 100 & 68.878 & 11.888 & 19.234 \\
\hline Providers & nc & 188 & 215 & 212 \\
\hline Visits with CDS requested (\%) & 25.211 & $30.859 \dagger^{*}$ & 22.86 & 6.442 \\
\hline Visits with CDS viewed (\%) & 24.271 & $29.700+^{*}$ & 20.73 & 7.023 \\
\hline Visits with CDS response (\%) & 23.493 & $28.669 \dagger^{*}$ & 12.55 & 6.932 \\
\hline
\end{tabular}

$\mathrm{nc}=$ not calculated, since a provider may have conducted visits in more than one of the three groups.

${ }^{*} P C P$ visit vs. Non-PCP visit pairwise comparison for each CDS behavior $p<0.001$

tType of visit vs CDS response $p<0.001$ across all three groups 


\section{References}

1. Starfield B, Shi L, Macinko J. Contribution of primary care to health systems and health. Milbank Q 2005; 83: 457-502.

2. Berwick DM, Nolan TW, Whittington J. The Triple Aim: Care, Health, And Cost. Health Aff 2008; 27: 759-769.

3. Osheroff JA, Teich JM, Middleton B, Steen EB, Wright A, Detmer DE. A Roadmap for National Action on Clinical Decision Support. J Am Med Inform Assoc 2007; 14: 141-145.

4. Garg AX, Adhikari NK, McDonald H, Rosas-Arellano MP, Devereaux PJ, Beyene J, Sam J, Haynes RB. Effects of computerized clinical decision support systems on practitioner performance and patient outcomes: A systematic review. JAMA 2005; 293(10): 1223-1238.

5. Bryan C, Boren SA. The use and effectiveness of electronic clinical decision support tools in the ambulatory/primary care setting: a systematic review of the literature. Inform Prim Care 2008; 16: 79-91.

6. Dexheimer JW, Talbot TR, Sanders DL, Rosenbloom ST, Aronsky D. Prompting clinicians about preventive care measures: a systematic review of randomized controlled trials. J Am Med Inform Assoc 2008; 15: $311-320$

7. Sequist TD, Gandhi TK, Karson AS, Fiskio JM, Bugbee D, Sperling M, Cook EF, Orav EJ, Fairchild DG, Bates DW. A Randomized Trial of Electronic Clinical Reminders to Improve Quality of Care for Diabetes and Coronary Artery Disease. J Am Med Inform Assoc 2005; 12: 431-437.

8. Shojania KG, Jennings A, Mayhew A, Ramsay CR, Eccles MP, Grimshaw J. The effects of on-screen, point of care computer reminders on processes and outcomes of care. Cochrane Database of Syst Rev 2009; 3.

9. van der Sijs H, Aarts J, Vulto A, Berg M. Overriding of Drug Safety Alerts in Computerized Physician Order Entry. J Am Med Inform Assoc 2006; 13: 138-147.

10. Sittig DF, Wright A, Osheroff JA, Middleton B, Teich JM, Ash JS, Campbell E, Bates DW. Grand challenges in clinical decision support. J Biomed Inform 2008; 41(2): 387-392.

11. Murphy DR, Reis B, Sittig DF, Singh H. Notifications Received by Primary Care Practitioners in Electronic Health Records: A Taxonomy and Time Analysis. Am J Med 2012; 125(2): 209.e1-209.e7.

12. Krall MA, Sittig DF. Clinician's assessments of outpatient electronic medical record alert and reminder usability and usefulness requirements. Proc AMIA Symp 2002; 400-404.

13. Medstar Health: Facts and Figures. Available at: http://www.medstarhealth.org/body.cfm?id=20. Accessed September 2, 2012.

14. Million Hearts - Home. Atlanta, GA: Centers for Disease Control and Prevention; c2012 [cited 2012 October 10]. Available from: http://millionhearts.hhs.gov/index.html.

15. Pham HH, Schrag D, O’Malley AS, Wu B, Bach PB. Care patterns in Medicare and their implications for pay for performance. N Engl J Med 2007; 356: 1130-1139.

16. Ferrante JM, Balasubramanian BA, Hudson SV, Crabtree BF. Principles of the Patient-Centered Medical Home and Preventive Services Delivery. Ann Fam Med 2010; 8: 108-116.

17. McClellan SR, Casalino LP, Shortell SM, Rittenhouse DR. When does adoption of health information technology by physician practices lead to use by physicians within the practice? J Am Med Inform Assoc 2013; 20: e26-e32.

18.Lanham HJ, Sittig DF, Leykum LK, Parchman ML, Pugh JA, McDaniel RR. Understanding differences in electronic health record (EHR) use: linking individual physicians' perceptions of uncertainty and EHR use patterns in ambulatory care. J Am Med Inform Assoc 2014; 21: 73-81.

19. McCormick D, Bor DH, Woolhandler S, Himmelstein DU. Giving Office-Based Physicians Electronic Access To Patients' Prior Imaging And Lab Results Did Not Deter Ordering Of Tests. Health Aff 2012; 31(3): 488-496. 
Appendix 1 PCP Characteristics at Visits

\begin{tabular}{|l|l|l|}
\hline Degree for PCP of patients & $\begin{array}{l}\text { Number } \\
(\mathbf{n = 6 8 , 7 1 4 )}\end{array}$ & Percent \\
\hline Physician patients & 62,432 & 90.858 \\
\hline Nurse Practitioner patients & 3,513 & 5.113 \\
\hline Physician Assistant patients & 1,567 & 2.280 \\
\hline Resident patients & 1,179 & 1.716 \\
\hline Other patients & 19 & 0.028 \\
\hline Nurse Midwife patients & 4 & 0.006 \\
\hline Specialty for PCP of patients & Number & Percent \\
\hline Internal Medicine & $\mathbf{( n = 6 8 , 7 2 2 )}$ & \\
\hline Family Medicine & 55,020 & 80.062 \\
\hline Medicine-Pediatrics & 12,834 & 18.675 \\
\hline Other & 618 & 0.899 \\
\hline
\end{tabular}

Appendix 2 Percent of PCP identification and continuity of care, by site

\begin{tabular}{|c|c|c|c|}
\hline & & $\begin{array}{l}\text { Visits } \\
\text { where a PCP is listed } \\
\text { (Percent by site) }\end{array}$ & $\begin{array}{l}\text { Visits } \\
\text { where the PCP is } \\
\text { seeing own patient } \\
\text { (Percent by site) }\end{array}$ \\
\hline \multicolumn{2}{|l|}{ Mean } & 76.665 & 62.387 \\
\hline \multicolumn{2}{|l|}{ Median } & 95.103 & 80.334 \\
\hline \multicolumn{2}{|c|}{ Std. Deviation } & 33.536 & 34.885 \\
\hline \multicolumn{2}{|l|}{ Range } & 99.17 & 99.36 \\
\hline \multicolumn{2}{|l|}{ Minimum } & 00.83 & 0 \\
\hline \multicolumn{2}{|l|}{ Maximum } & 1.0000 & 99.36 \\
\hline \multirow[t]{3}{*}{ Percentiles } & 25 & 67.713 & 35.665 \\
\hline & 50 & 95.103 & 80.334 \\
\hline & 75 & 99.050 & 90.948 \\
\hline
\end{tabular}

